\title{
Diversidad genética y estructura poblacional de Anopheles triannulatus s.I. en Córdoba, Colombia, determinadas mediante el método de región de código de barras de ADN
}

\author{
María Atencia-Pineda, Angie Toro-Cantillo, Richard Onalbi Hoyos-López \\ Grupo de Investigaciones en Enfermedades Tropicales y Resistencia Bacteriana, \\ Universidad del Sinú, Montería, Colombia
}

Introducción. A pesar de los recientes reportes de infección con Plasmodium spp. en poblaciones relacionadas con los linajes noroeste y sureste, Anopheles triannulatus no está incriminado como vector de la transmisión de malaria en Colombia. La diversidad genética puede delimitar la información sobre el flujo génico y la diferenciación poblacional entre localidades con malaria.

Objetivo. Estimar la diversidad genética de An. triannulatus en cinco municipios con alta y baja incidencia de malaria en el departamento de Córdoba.

Materiales y métodos. La recolección entomológica se hizo entre agosto y noviembre de 2016 en los municipios de Tierralta, Puerto Libertador, Montelíbano, Sahagún y Planeta Rica. Como marcador genético, se utilizó la región de código de barras de ADN del gen mitocondrial $C O I$. El análisis genético incluyó la estimación de los parámetros de diversidad haplotípica, estructura genética y flujo génico, la prueba $\mathrm{D}$ de neutralidad de Tajima, la red de haplotipos y las relaciones filogenéticas.

Resultados. Se obtuvieron 148 secuencias parciales de 655 nucleótidos del gen $\mathrm{COI}$, de los cuales se derivaron 44 haplotipos. Los haplotipos $\mathrm{H} 2$ y H21 fueron los más frecuentes en las poblaciones. Los valores de la prueba $D$ de Tajima fueron negativos y no significativos $(p>0,10)$. Los estimadores de estructura genética $\left(\mathrm{F}_{\mathrm{ST}}=0,01427\right)$ y de flujo génico $(\mathrm{Nm}=17,27)$ evidenciaron que no hubo diferenciación genética en las poblaciones muestreadas debido al importante intercambio de migrantes. Mediante las inferencias filogenéticas y la red de haplotipos, se identificó una sola especie sin diferenciación geográfica o de linajes en el rango geográfico estudiado.

Conclusión. La diversidad genética calculada para An. triannulatus en este contexto, indicó que las poblaciones están en un intercambio constante.

Palabras clave: Anopheles/genética; variación genética; haplotipos; flujo génico; Colombia.

doi: https://doi.org/10.7705/biomedica.v38i0.4055

Genetic diversity and population structure of Anopheles triannulatus s. I. in the department of Córdoba, Colombia, using DNA barcoding

Introduction: Anopheles triannulatus is not incriminated as a vector of malaria transmission in Colombia despite recent reports of infection with Plasmodium spp. in populations related to the northwestern and southeastern lineages. Genetic diversity can delimit information about gene flow and population differentiation in localities with malaria.

Objective: To estimate the genetic diversity of An. triannulatus in five municipalities with high and low incidence of malaria in the department of Córdoba.

Materials and methods: The entomological collections were done between August and November, 2016, in Tierralta, Puerto Libertador, Montelíbano, Sahagún, and Planeta Rica. We used the COI barcoding fragment as molecular marker. The genetic analysis included the estimation of genetic parameters such as the diversity haplotype, the genetic structure, the gene flow, the Tajima's D test, the haplotype network, and the phylogenetic relationship.

Results: We obtained 148 sequences with a length of 655 nucleotides of the $\mathrm{COI}$ gene, from which we derived 44 haplotypes. The $\mathrm{H} 2$ and $\mathrm{H} 21$ haplotypes were the most frequent in the populations. The values of the Tajima's $D$ test were negative and not significant $(p>0.10)$. The genetic structure index $\left(F_{S T}=0.01427\right)$ and the gene flow $(\mathrm{Nm}=17.27)$ evidenced no differentiation between sampled

\section{Contribución de los autores:}

Maria Atencia-Pineda: experimentos y trabajo de campo, análisis de los datos y escritura del manuscrito

Angie Onalbi Toro-Cantillo: experimentos y trabajo de campo

Richard Onalbi Hoyos-López: diseño del estudio, análisis de los datos y escritura del manuscrito 
populations due to the high exchange of migrants. Using phylogenetic inferences and the haplotype network, we identified one single species without geographic differentiation or lineages in the geographic range studied.

Conclusions: The genetic diversity calculated for An. triannulatus in this context indicated stable populations in constant exchange.

Key words: Anopheles/genetics, genetic variation; haplotypes; gene flow; Colombia. doi: https://doi.org/10.7705/biomedica.v38i0.4055

Anopheles triannulatus (Neiva y Pinto, 1922) pertenece al grupo Oswaldoi del subgénero Nysorhynchus, y se encuentra distribuido en Argentina, Bolivia, Brasil, Colombia, Costa Rica, Ecuador, Guayana Francesa, Guatemala, Guyana, Nicaragua, Panamá, Paraguay, Perú, Puerto Rico, Surinam, Trinidad y Tobago, y Venezuela (1-3).

En Colombia, esta especie se distribuye en 29 de los 32 departamentos (4). Su importancia como vector en la transmisión de la malaria o paludismo es relativa, pues se basa en evidencia circunstancial o en la identificación de la proteína del circumsporozoíto (Circumsporozoite Protein, CSP) de Plasmodium vivax (genotipos VK210 y VK247), Plasmodium falciparum y Plasmodium malariae en hembras $(5,6)$. Se ha considerado como un vector secundario local de la malaria en varias zonas de Brasil $(7,8)$ y como vector dominante en el este de Loreto, Perú (9). Recientemente, se detectó An. triannulatus infectado con el genotipo VK247 de $P$. vivax en El Bagre, Antioquia, y con $P$. falciparum en Puerto Libertador, Córdoba, con una abundancia significativa de la especie en este último sitio (28\% del total de Anopheles spp.) $(10,11)$. Sin embargo, a pesar de los reportes de infección natural con especies de Plasmodium, en Colombia no se ha incriminado como vector.

Anopheles triannulatus se considera un complejo de tres especies (Anopheles triannulatus s.s., Anopheles halophylus y Anopheles triannulatus C) por la amplia diversidad morfológica en los genitales masculinos, los huevos y las larvas. Además, se han reportado algunas diferencias en los patrones de distribución, en su ecología y su comportamiento (12-14). Asimismo, se conocen algunos resultados y datos moleculares sobre aloenzimas que permitieron diferenciar entre An. halophylus y $A n$.

\section{Correspondencia:}

Richard Onalbi Hoyos, Laboratorio de Investigaciones en Ciencias Biomédicas y Biología Molecular, Universidad del Sinú, carrera 1w №38-153, Montería, Colombia

Telefax: (574) 7841961

rhoyoslopez@gmail.com

Recibido: 01/09/17; aceptado: 12/06/18 triannulatus (15) con base en el gen intemporal y el gen CPR; con el primero, no se pudieron diferenciar las tres especies que conforman el complejo An. triannulatus, en tanto que con el segundo se diferenciaron claramente (16). Esta amplia diversidad implicaría diferencias en la transmisión de Plasmodium spp. y en el comportamiento de picadura (antropofílico y zoófilo) según la distribución geográfica, como se ha propuesto para otras especies (17-20). En Colombia, solo se ha reportado la presencia de $A n$. triannulatus s.l., pero las otras dos especies que conforman el complejo no se han encontrado en el país $(10,21)$.

En Colombia, hay pocos estudios de genética de poblaciones y estructura genética de $A n$. triannulatus s.l. encaminados a resolver el estatus taxonómico de la especie, lo cual permite estudiar los linajes y especies del complejo. En este sentido, el estudio de Rosero, et al. (21), es el más relevante, ya que los autores diferenciaron dos linajes (sureste y noroccidente) con flujo génico limitado y una estructura genética divergente con base en las secuencias del COIy del ITS2 (Internal Transcribed Spacer 2).

En esta investigación, los especímenes de $A n$. triannulatus recolectados en Puerto Libertador, se agruparon en el clado noroccidente y se relacionaron filogenéticamente con individuos del Bajo Cauca (EI Bagre) y el Urabá antioqueño (San Pedro de Urabá), y en el linaje sureste se agruparon los individuos recolectados en los departamentos del Meta y Amazonas (Leticia, Puerto Nariño y Tarapacá) (11), linajes que concuerdan con su ubicación geográfica.

A pesar de este hallazgo, persisten algunos vacíos, pues el estudio se restringió a cuatro departamentos y, en el caso del departamento de Córdoba (con muchas áreas de alta y baja endemia para malaria), solo se estudió la población de Puerto Libertador. Por ello, sería de gran importancia ampliar la recolección a todos los departamentos y municipios donde se encuentra distribuida la especie, para recabar información sobre el estatus real de An. triannulatus en Colombia. Además, se 
requieren otras herramientas moleculares para complementar el estudio de la morfología de los estadios inmaduros, los genitales de los machos y la morfometría geométrica, con el fin de acopiar información confirmada sobre este complejo en el país.

En objetivo del presente estudio fue establecer cómo se relacionaban las poblaciones de $A n$. triannulatus de tres municipios endémicos para malaria y otros dos no endémicos en el departamento de Córdoba, estimando la distribución y la diversidad de haplotipos, calculando el flujo génico y haciendo la diferenciación genética de las poblaciones muestreadas, así como la relación filogenética de los individuos recolectados en el departamento de Córdoba con los de otras áreas de Colombia mediante el método de código de barras (barcoding) de ADN del gen de la citocromo oxidasa I (COI).

\section{Materiales y métodos}

\section{Recolección y procesamiento de los mosquitos}

Las recolecciones se hicieron entre agosto y noviembre del 2016 en cinco municipios del departamento de Córdoba utilizando trampas Shannon y CDC durante tres días, entre las 18:00 y las 00:00 horas (figura 1) (cuadro1). La identificación taxonómica de las hembras de An. triannulatus s.l. se basó en la clave dicotómica para la identificación de anofelinos en Colombia (4). EI ADN se extrajo del abdomen del insecto utilizando el método de altas concentraciones de sales descrito por Collins, et al. (22), modificado por Atencia, et al. (23).

\section{Amplificación de la región de código de barras del gen $\mathrm{COI}$}

En la reacción en cadena de la polimerasa (PCR) se utilizaron los cebadores universales LCO1490: 5'-GGTCAACAAATCATAAAGATATTGG-3' y HCO 2198: 5'-TAAACTTCAGGGTGACCAAAAAATCA-3', en un volumen final de $30 \mu \mathrm{l}$ y usando $1,5 \mathrm{mM}$ de $\mathrm{MgCl}_{2}, 0,2 \mathrm{mM}$ de cada dNTP, 0,3 $\mu \mathrm{M}$ de cada cebador, $1 \mathrm{X}$ de solución tampón (con 1,5 mM de $\mathrm{MgCl}_{2}$ ), 2 unidades de Taq polimerasa (TopTaq ${ }^{\mathrm{TM}}$, Qiagen) y $2 \mu \mathrm{l}$ de ADN, todo ajustado con agua ultrapura estéril (24).

El perfil térmico consistió en un primer ciclo de desnaturalización a $95{ }^{\circ} \mathrm{C}$ durante cinco minutos, seguido de 35 ciclos de desnaturalización durante 30 segundos a $94{ }^{\circ} \mathrm{C}$, un alineamiento a $46{ }^{\circ} \mathrm{C}$ durante un minuto, una extensión a $72{ }^{\circ} \mathrm{C}$ durante un minuto y un paso final de extensión a $72{ }^{\circ} \mathrm{C}$

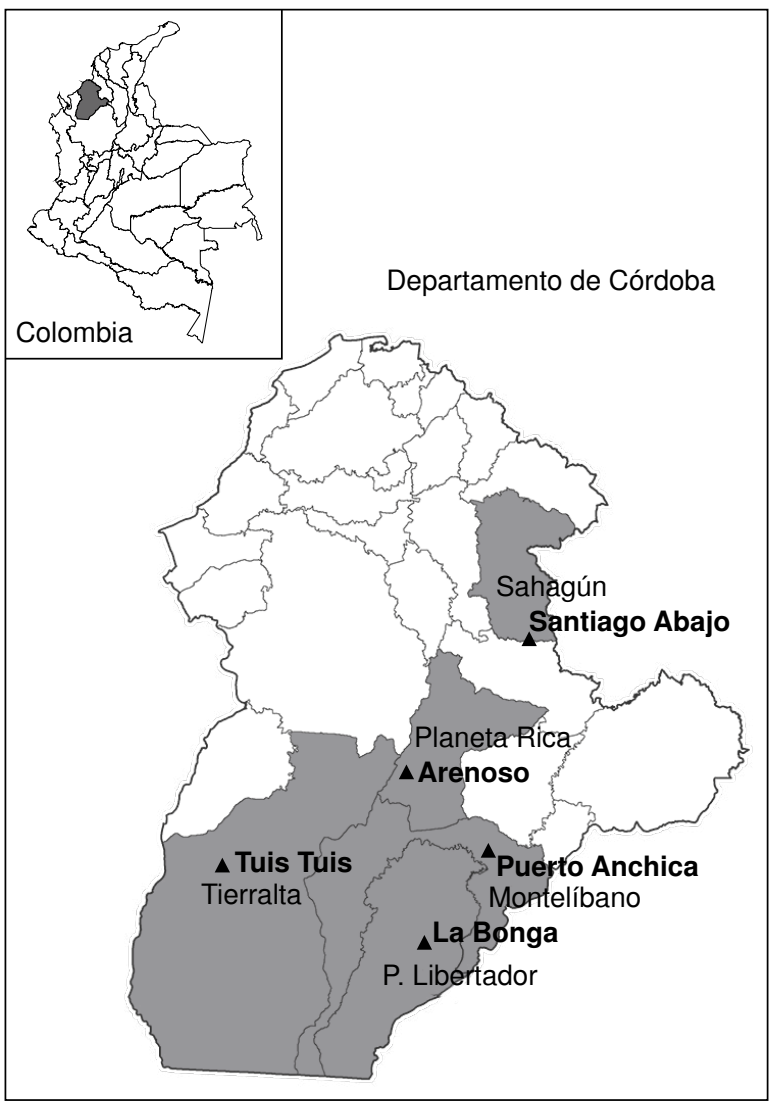

Figura 1. Distribución de las localidades de recolección de Anopheles triannulatus s.l. en el departamento de Córdoba, Colombia

Cuadro 1. Sitios de recolección y número de individuos recolectados ( $n$ ) de Anopheles triannulatus s. I. en el departamento de Córdoba, Colombia

\begin{tabular}{lcc}
\hline Municipio, localidad & n & $\begin{array}{c}\text { Coordenadas } \\
\text { (latitud, longitud) }\end{array}$ \\
\hline Tierralta, Tuis Tuis & 76 & $7,95112-76,25753$ \\
Puerto Libertador, La Bonga & 25 & $7,73731-75,68668$ \\
Montelíbano, Puerto Anchica & 25 & $7,99530-75,50817$ \\
Sahagún, Santiago Abajo & 11 & $8,58783-75,39235$ \\
Planeta Rica, Arenoso & 11 & $8,21764-75,73914$ \\
\hline
\end{tabular}

durante 10 minutos. Los productos amplificados, teñidos con bromuro de etidio, se visualizaron mediante electroforesis en gel de agarosa al $1 \%$. La secuenciación de los productos de la PCR se hizó con el sistema de Macrogen, Inc., en un secuenciador ABI PRISM ${ }^{\mathrm{TM}}$ (Applied Biosystems).

\section{Análisis de los datos}

Las secuencias obtenidas se editaron y alinearon con el programa MEGA, versión 7.0 (25) y, el alineamiento múltiple, con el programa CLUSTAL 
W (26). A cada secuencia de consenso se le hizo el alineamiento con el programa BLASTn (27), con el fin de comparar las secuencias de An. Triannulatus s.l. almacenadas en el GenBank y en Barcode of Life Data System (Bold Systems) (28).

El número de haplotipos y su frecuencia, el número de sitios polimórficos (S), el número promedio de diferencias de nucleótidos (k), la diversidad de haplotipos $(\mathrm{DH})$ y la diversidad de nucleótidos $(\pi)$, la prueba $D$ de neutralidad de Tajima y la distribución de diferencias (mismatch), se calcularon con el programa DnaSP, versión 5 (29). Los estimadores de estructura genética $\left(F_{S T}\right)$ y de flujo génico $(\mathrm{Nm})$ se estimaron en el programa Arlequin, versión 3.5 (30).

\section{Red de haplotipos y análisis filogenético}

La red estimada entre los haplotipos caracterizados se graficó con el programa Network, versión 5.0 (31) y el algoritmo median-joining (32). El modelo evolutivo se infirió con base en los criterios de información Akaike (AIC) incluidos en el programa jModelTest, versión 2 (33). Esta información se utilizó para la inferencia filogenética bayesiana con el programa MrBayes, versión 3.2 (34).

Con la cadena de Markov-Monte-Carlo se obtuvieron 10 millones de generaciones. Para la construcción del árbol se utilizaron secuencias de $A n$. triannulatus s.l. de Colombia reportadas en el GenBank: JX205111.1 -JX205113.1, HM022387.1HM022389.1 y KY921784.1.

\section{Resultados}

Análisis de la región de código de barras del COI

Se recolectaron 148 hembras de An. triannulatus

s.l. en las cinco localidades muestreadas (cuadro
1). La longitud de las secuencias del gen $\mathrm{COI}$ obtenidas de cada individuo fue de 655 nucleótidos, con un contenido promedio de A+T de $69 \%$. Se encontraron 44 haplotipos mitocondriales del gen COI (GenBank NA MF043261 a MF043304) (cuadro 2). Las secuencias presentaron 620 sitios conservados y 35 posiciones variables (21 sitios fueron parsimoniosamente informativos $y$ 14 sitios de mutaciones únicas); se distinguieron 158 transiciones y 9 transversiones (cuadro 3). Las distancias genéticas intraespecíficas alcanzaron un valor máximo de 0,016, observado entre los pares de los haplotipos $\mathrm{H} 32-\mathrm{H} 40$ y H37-H40.

\section{Diversidad genética, prueba de neutralidad y estructura genética}

Los valores de los índices de diversidad genética y la prueba de neutralidad para las cinco poblaciones de An. triannulatus s.l. se muestran en el cuadro 4. Los promedios más altos en $\mathrm{K}, \mathrm{DH}$ у п fueron los correspondientes a La Bonga (Puerto Libertador) y Santiago Abajo (Sahagún). La prueba de neutralidad arrojó valores negativos, con excepción de los especímenes de Arenoso (Planeta Rica), en los que fue positiva, pero no significativa $(p>0,10)$. La distribución de diferencias fue unimodal, es decir, que había expansión poblacional (figura 2). Las distancias genéticas entre las poblaciones fueron bajas, en un rango de 0,00387 a 0,00516.

En general, los valores pareados de la estructura genética fueron de $\mathrm{F}_{\mathrm{ST}}=0,01427$ y los del flujo génico de $\mathrm{Nm}=17,27$ para todas las poblaciones muestreadas, lo cual implica una diferenciación genética nula entre las poblaciones y un elevado intercambio de migrantes (cuadro 5).

Cuadro 2. Número y frecuencia de haplotipos de Anopheles triannulatus s.I. en cinco poblaciones del departamento de Córdoba, Colombia

\begin{tabular}{llr}
\hline Municipio y localidad & Haplotipos (frecuencia) & $\begin{array}{c}\text { Total de } \\
\text { haplotipos }\end{array}$ \\
\hline Tierralta, Tuis Tuis & $\mathrm{H} 1(2), \mathrm{H} 2(21), \mathrm{H} 3(5), \mathrm{H} 4(5), \mathrm{H} 5(1), \mathrm{H} 6(1), \mathrm{H} 7(1), \mathrm{H} 8(3), \mathrm{H} 9(1), \mathrm{H} 10(2), \mathrm{H} 11(1)$, \\
& $\mathrm{H} 12(3), \mathrm{H} 13(3), \mathrm{H} 14(1), \mathrm{H} 15(1), \mathrm{H} 16(4), \mathrm{H} 17(2), \mathrm{H} 18(1), \mathrm{H} 19(4), \mathrm{H} 20(1), \mathrm{H} 21(4)$, & 29 \\
& $\mathrm{H} 22(1), \mathrm{H} 23(2), \mathrm{H} 24(1), \mathrm{H} 25(1), \mathrm{H} 26(1), \mathrm{H} 27(1), \mathrm{H} 28(1), \mathrm{H} 29(1)$ \\
Puerto Libertador, La Bonga & $\mathrm{H} 2(5), \mathrm{H} 3(3), \mathrm{H} 13(1), \mathrm{H} 16(3), \mathrm{H} 21(4), \mathrm{H} 23(1), \mathrm{H} 30(1), \mathrm{H} 31(1), \mathrm{H} 32(1), \mathrm{H} 33(1), \mathrm{H} 34(1)$, & 14 \\
& $\mathrm{H} 35(1), \mathrm{H} 36(1), \mathrm{H} 37(1)$. & \\
Montelíbano, Puerto Anchica & $\mathrm{H} 2(5), \mathrm{H} 3(3), \mathrm{H} 4(2), \mathrm{H} 13(1), \mathrm{H} 17(1), \mathrm{H} 21(8), \mathrm{H} 38(1), \mathrm{H} 39(1), \mathrm{H} 40(1), \mathrm{H} 41(1), \mathrm{H} 42(1)$. \\
Sahagún, Santiago Abajo & $\mathrm{H} 1(1), \mathrm{H} 2(2), \mathrm{H} 3(3), \mathrm{H} 13(1), \mathrm{H} 17(1), \mathrm{H} 21(1), \mathrm{H} 23(1), \mathrm{H} 43(1)$, \\
Planeta Rica, Arenoso & $\mathrm{H} 2(4), \mathrm{H} 3(2), \mathrm{H} 4(1), \mathrm{H} 21(3), \mathrm{H} 44(1)$. \\
Total & $\mathrm{H} 1(3), \mathrm{H} 2(37), \mathrm{H} 3(16), \mathrm{H} 4(8), \mathrm{H} 5(1), \mathrm{H} 6(1), \mathrm{H} 7(1), \mathrm{H} 8(3), \mathrm{H} 9(1), \mathrm{H} 10(2), \mathrm{H} 11(1)$, & 8 \\
& $\mathrm{H} 12(3), \mathrm{H} 13(6), \mathrm{H} 14(1), \mathrm{H} 15(1), \mathrm{H} 16(7), \mathrm{H} 17(4), \mathrm{H} 18(1), \mathrm{H} 19(4), \mathrm{H} 20(1), \mathrm{H} 21(20)$, & 4 \\
& $\mathrm{H} 22(1), \mathrm{H} 23(4), \mathrm{H} 24(1), \mathrm{H} 25(1), \mathrm{H} 26(1), \mathrm{H} 27(1), \mathrm{H} 28(1), \mathrm{H} 29(3), \mathrm{H} 30(1), \mathrm{H} 31(1)$, & 44 \\
& $\mathrm{H} 32(1), \mathrm{H} 33(1), \mathrm{H} 34(1), \mathrm{H} 35(1), \mathrm{H} 36(1), \mathrm{H} 37(1), \mathrm{H} 38(1), \mathrm{H} 39(1), \mathrm{H} 40(1), \mathrm{H} 41(1)$, & \\
\hline
\end{tabular}


Cuadro 3. Sitios variables en la secuencia del gen $\mathrm{COI}$ de 44 haplotipos de Anopheles triannulatus s.l. Los puntos (.) denotan homología de nucleótidos.

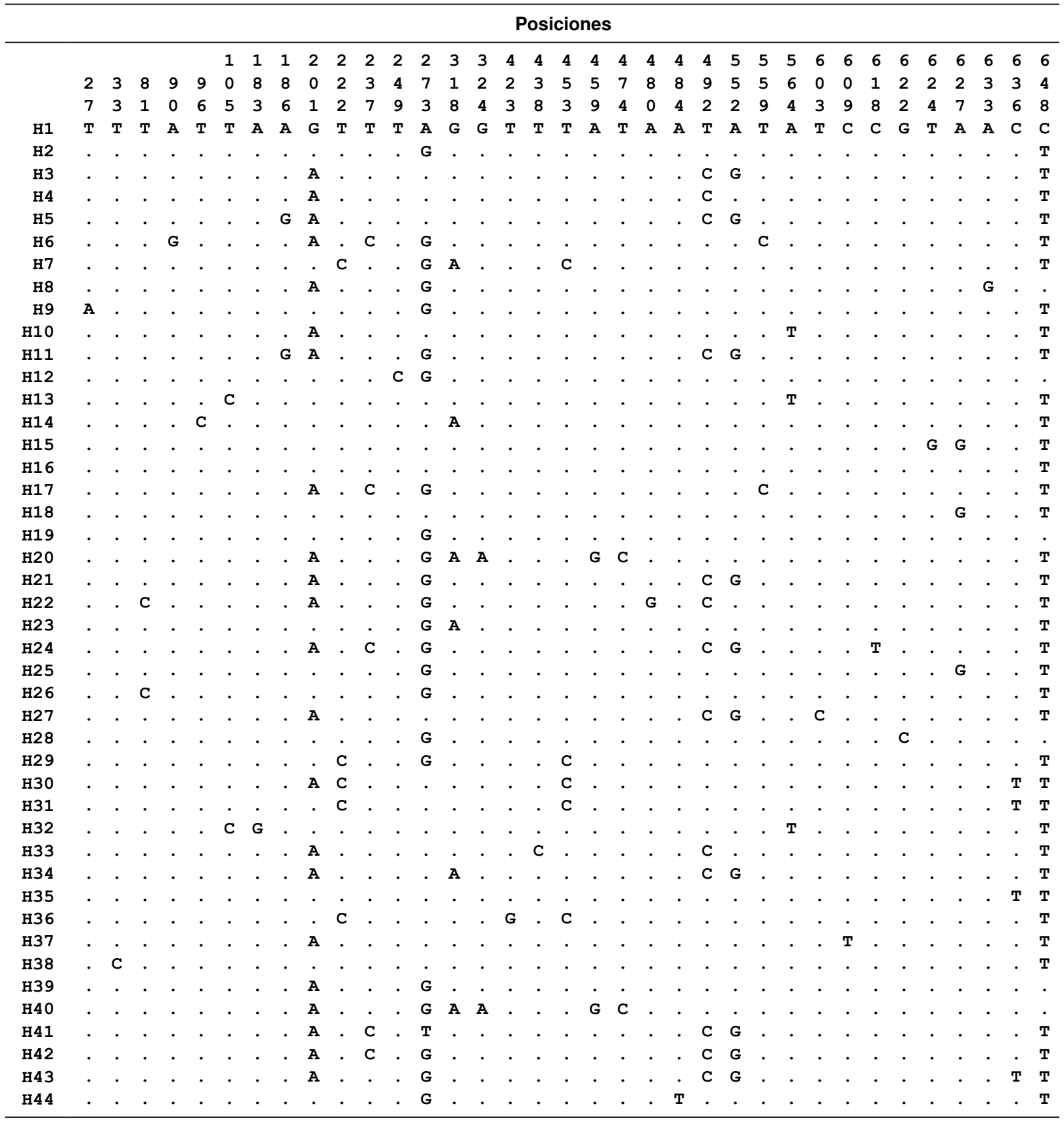

\section{Red de haplotipos y análisis filogenético}

En la figura 3 se muestra la red inferida para las poblaciones de An. triannulatus s.l. El torso de la red está estructurado por los haplotipos 1, 2, 3, 4, $5,7,10,11,16,18,19,21,23,25,29,31$ y 35 ; los haplotipos más frecuentes fueron $\mathrm{H} 2(25 \%)$ y H21 $(13,5 \%)$. Estos dos haplotipos se establecieron en todas las poblaciones. El árbol filogenético obtenido con inferencia bayesiana evidenció un único clado monofilético para todos los haplotipos caracterizados de An. triannulatus s.I. en Córdoba; la segregación geográfica no se evidenció o no se definieron linajes dentro del clado estimado (figura 4).

\section{Discusión}

Según la inferencia filogenética bayesiana efectuada en el presente estudio, los ejemplares de An. triannulatus s.l. en Córdoba correspondían 
Cuadro 4. Parámetros de diversidad genética y prueba de neutralidad en cinco poblaciones de Anopheles triannulatus s.l. del departamento de Córdoba, Colombia. NH: número de haplotipos en cada población; S: sitios polimórficos; K: número promedio de divergencias nucleotídicas; $\mathrm{DH}$ : diversidad de haplotipos; $\pi$ : diversidad nucleotídica. En la prueba de neutralidad no se encontraron diferencias estadísticas $(\mathrm{p}>0,10)$.

\begin{tabular}{|c|c|c|c|c|c|c|c|}
\hline Municipio & Localidad & NH & $\mathbf{S}$ & $\mathbf{K}$ & DH & $\pi$ & Tajima's D \\
\hline Tierralta & Tuis Tuis & 29 & 28 & 3,11404 & 0,90842 & 0,00475 & -1.42043 \\
\hline Puerto Libertador & La Bonga & 14 & 14 & 3,38667 & 0,92667 & 0,00517 & -0.30050 \\
\hline Montelíbano & Puerto Anchica & 11 & 14 & 2,93000 & 0,86000 & 0,00447 & -0.91886 \\
\hline Sahagún & Santiago Abajo & 8 & 11 & 3,45455 & 0,92727 & 0,00517 & -0.34492 \\
\hline Planeta Rica & Arenoso & 5 & 5 & 2,25455 & 0,81818 & 0,00344 & 1.22875 \\
\hline Total & & 44 & 35 & 3.18174 & 0,90182 & 0,00486 & $-1,50819$ \\
\hline
\end{tabular}

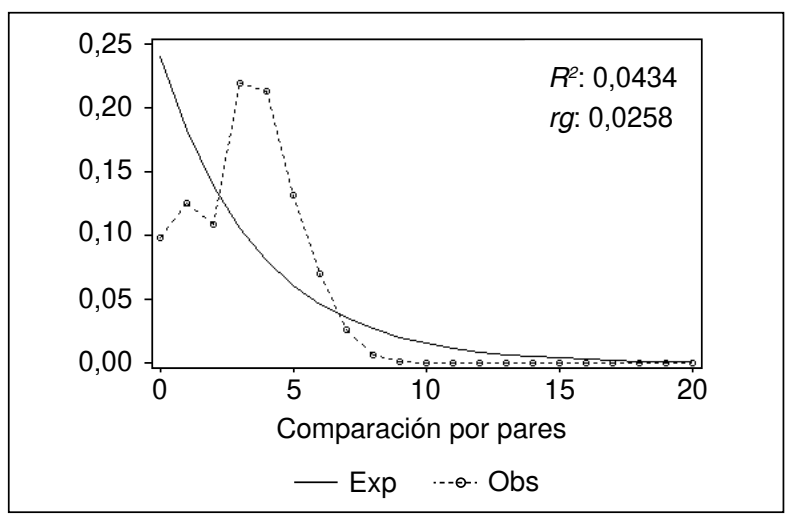

Figura 2. Gráfica de distribución de diferencias (mismatch) entre haplotipos de Anopheles triannulatus s.l. de cinco poblaciones del departamento de Córdoba, Colombia

Cuadro 5. Distancia genética y flujo génico basados en los valores $\mathrm{F}_{\mathrm{ST}}$ (arriba de la diagonal), el índice de fijación y el $\mathrm{Nm}$ (abajo de la diagonal) del número de migrantes efectivos en cinco poblaciones de Anopheles triannulatus s. I.

\begin{tabular}{lccccc}
\hline Localidad & $\begin{array}{c}\text { Tuis } \\
\text { Tuis }\end{array}$ & La Bonga & $\begin{array}{c}\text { Puerto } \\
\text { Anchica }\end{array}$ & $\begin{array}{c}\text { Santiago } \\
\text { Abajo }\end{array}$ & Arenoso \\
\hline Tuis Tuis & - & 0,031 & 0,093 & 0,011 & 0,035 \\
La Bonga & 15,386 & - & 0,066 & $-0,031$ & 0,015 \\
$\begin{array}{l}\text { Puerto } \\
\text { Anchica }\end{array}$ & 4,856 & 7,048 & - & $-0,032$ & $-0,037$ \\
$\begin{array}{l}\text { Santiago } \\
\text { Abajo }\end{array}$ & 46,211 & inf & inf & - & $-0,056$ \\
Arenoso & 13,889 & 31,869 & inf & inf & - \\
\hline
\end{tabular}

exclusivamente a un linaje monofilético agrupado en un clado. Los datos del código de barras evidenciaron una diferenciación genética nula entre las poblaciones sometidas a prueba, pertenecientes a áreas con una mayor transmisión de malaria en el contexto microgeográfico para el departamento de Córdoba.

En esta área se han establecido patrones similares para otras especies como An. darlingi (35), An. albimanus (19) y An. nuneztovari s.l. (36). Todas estas especies mostraron una gran diversidad genética homogénea en este nivel geográfico, donde no hay aislamiento por distancia o segregación genética (figura 4). Curiosamente, a pesar de las barreras importantes que son los ríos Sinú y San Jorge, y de las diferencias ecológicas y climáticas entre las zonas muestreadas (bosque húmedo tropical en Tierralta y Puerto Libertador, y bosque seco tropical en Montelíbano, Planeta Rica y Sahagún), Tierralta, Puerto Libertador y Montelíbano son localidades con gran endemia de malaria, y hubo pocos o ningún caso en Sahagún y Planeta Rica.

La diversidad de haplotipos fue significativa y la red permitió observar muchos compartidos por todas las localidades, lo que sugiere una historia evolutiva similar para esta especie en el departamento de Córdoba (figura 3). Otra evidencia de esta hipótesis es la agrupación de especímenes recolectados en Puerto Libertador con poblaciones de Antioquia (El Bagre, Zaragoza y San Pedro de Urabá), lo cual estructuraría el linaje noroccidente para An. triannulatus s.l. con base en marcadores moleculares como el COI y el ITS2 (21).

Los valores estimados para $\mathrm{F}_{\mathrm{ST}}$ y $\mathrm{Nm}$ sugieren un gran flujo génico entre las poblaciones muestreadas y una estructura genética nula; en este sentido, la diversidad genética de haplotipos y los valores de la prueba $\mathrm{D}$ de Tajima corresponden a una expansión de la población y no a la presión selectiva, lo cual implica que An. triannulatus s.l. comprende una sola población; estos resultados son similares a los de poblaciones de An. triannulatus s.l. estudiadas con base en secuencias del $\mathrm{COI}$ (no código de barras) en el río Amazonas, y el centro y el sur de Brasil (37). En Colombia, la distribución desigual de los linajes noroccidente y sureste evidenció una distribución bimodal, lo cual sugiere un cuello de botella o eventos de colonización entre las localidades muestreadas (21). 


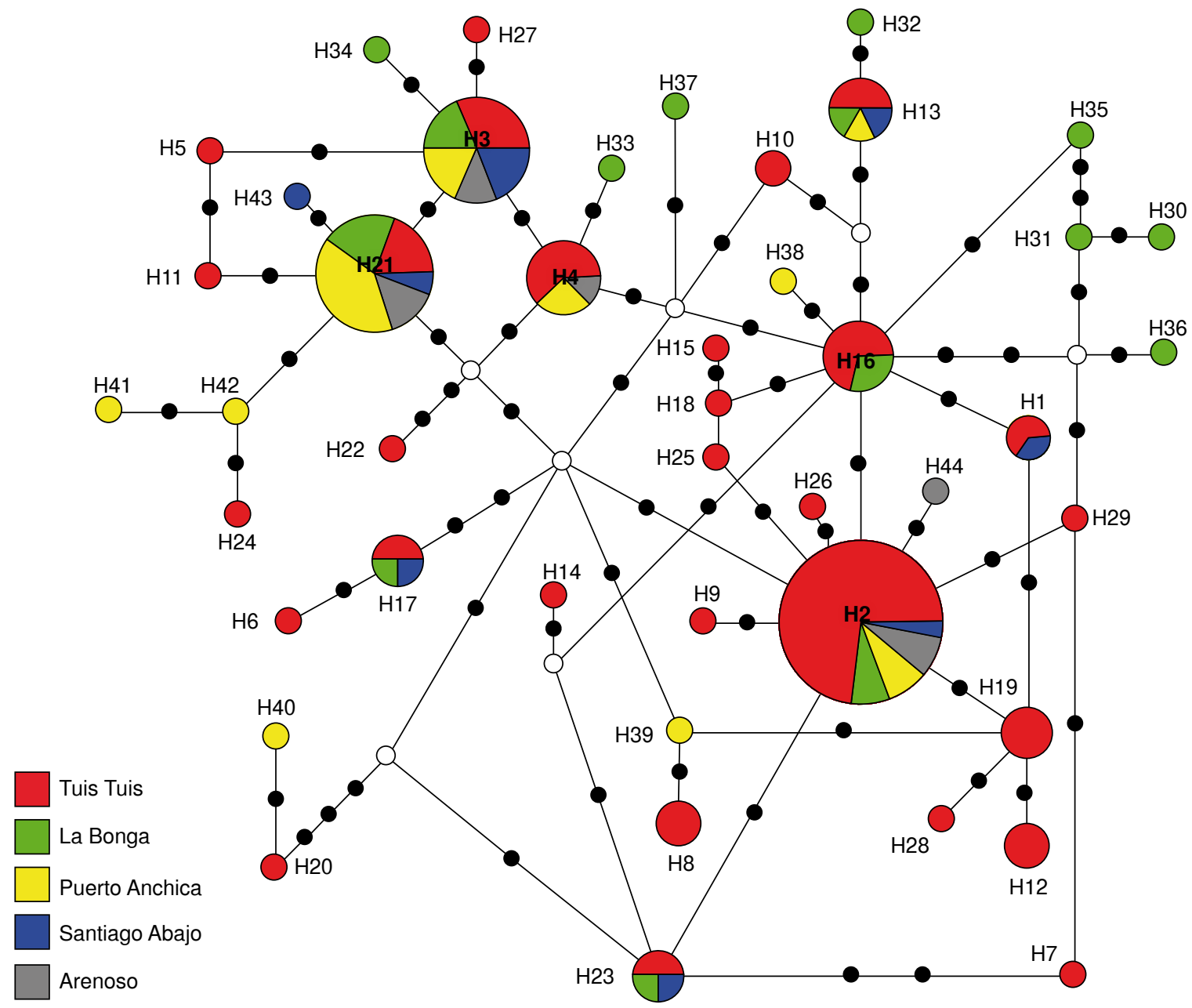

Figura 3. Red de haplotipos inferida con las secuencias del gen COI de 148 individuos de Anopheles triannulatus s.I. El área de cada círculo es proporcional a la frecuencia del haplotipos. Los círculos negros representan los pasos de mutación y, los círculos blancos, los haplotipos desaparecidos o no muestreados. La fracción de cada círculo indica la frecuencia de los haplotipos presentes en las poblaciones estudiadas.

La expansión poblacional evidenciada por la prueba $\mathrm{D}$ de Tajima y la distribución de diferencias podría deberse a la presencia de haplotipos únicos en cada una de las poblaciones muestreadas, lo cual coincide con una gran diversidad genética $(\mathrm{DH}=0,90182)$. Valores similares se han registrado en especies como Anopheles punctimacula s.l. (DH: 0,6444 y 0,7222) (38), Anopheles sinensis (DH, 0,99) (39), Anopheles nuneztovari (40) y Anopheles marajoara de linaje $1(\mathrm{DH}: 0,828)$ y de linaje 2 (DH: 0,780) (41).

Algunos de los haplotipos encontrados en este estudio ya fueron reportados en un trabajo realizado en Córdoba por Ahumada, et al., en el cual los haplotipos $\mathrm{H} 2, \mathrm{H} 19, \mathrm{H} 21, \mathrm{H} 25$ y $\mathrm{H} 43$ se nombraron como haplotipo 2; los $\mathrm{H} 8$ y H39, como haplotipo 8; el $\mathrm{H} 10$, como haplotipo 15; el $\mathrm{H} 17$, como haplotipo 6, y los $\mathrm{H} 23$ y H28, como haplotipo 3 (42). Sin embargo, es importante destacar que las secuencias reportadas por estos autores registraron una longitud de 607 nucleótidos, cuyo primer nucleótido tiene correspondencia con el nucleótido 6 y, el último, con el de la posición 612 de las secuencias obtenidas en este estudio, razón por la cual un haplotipo de los reportados por ellos es similar a varios de los de este estudio (42).

El árbol filogenético evidenció dos clados: uno conformado por secuencias de individuos pertenecientes al municipio del Meta y el otro formado por dos subclados correspondientes a las secuencias de Córdoba y de Turbo, Antioquia, y el otro, por secuencias de Putumayo, aunque con un soporte de rama bajo; estos resultados también coinciden con los obtenidos por Rosero, et al. (21). 


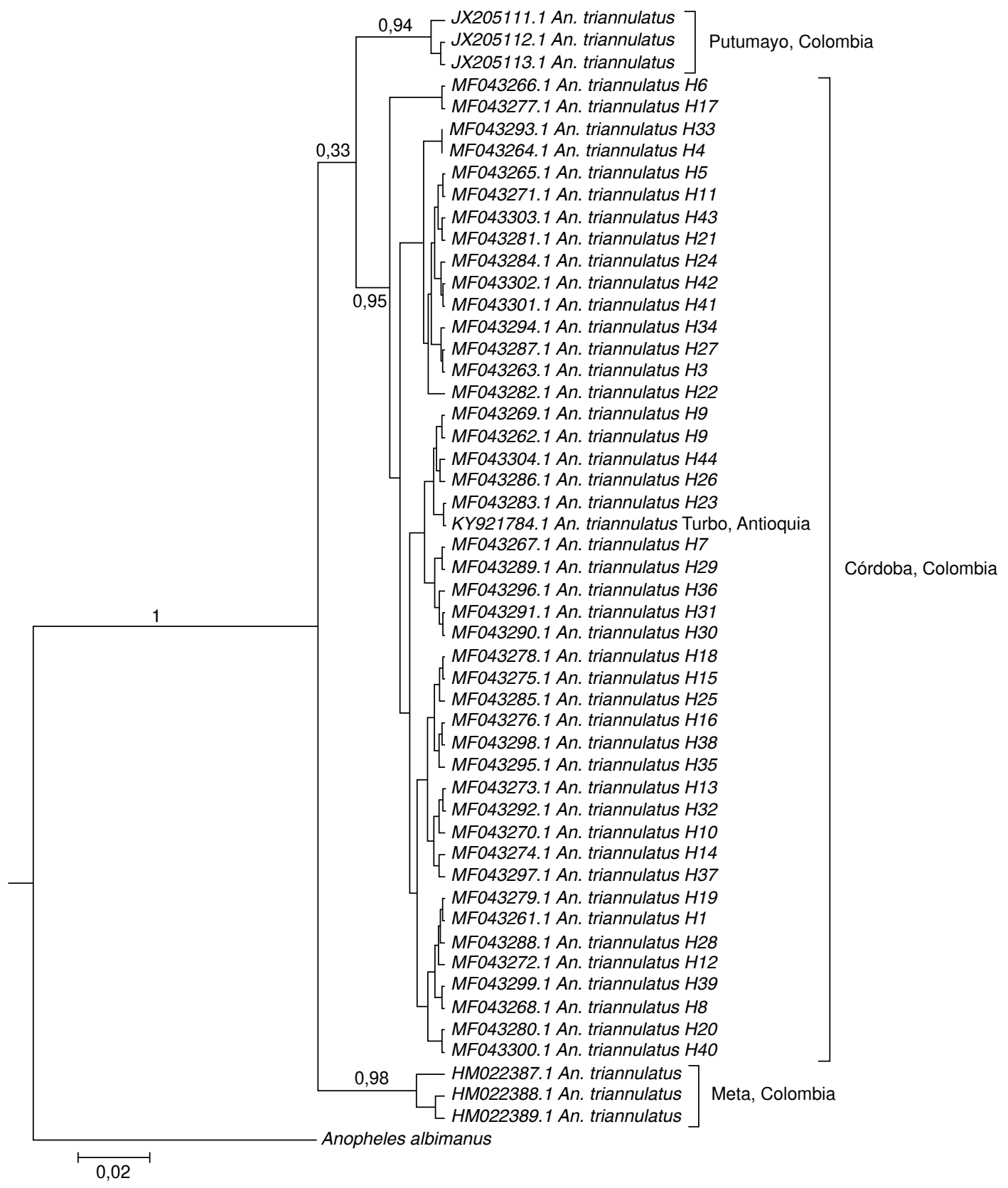

Figura 4. Árbol de inferencia filogenética bayesiana según el modelo de evolución molecular HKY + G + I con secuencias del gen $\mathrm{COI}$ caracterizado en cinco poblaciones de An. Triannulatus s. I. del departamento Córdoba, Colombia. El número sobre las ramas representa las probabilidades posteriores del clado.

Puede observarse que las secuencias de Córdoba y de Turbo, Antioquia, pertenecerían al linaje noroccidente $y$, las secuencias de Meta, al linaje sureste. Sin embargo, como ya se mencionó, el subclado correspondiente a las secuencias de Putumayo, que por ubicación geográfica debería haberse agrupado con los individuos del Meta para formar el clado sureste, se agrupó en un clado independiente con las secuencias de Córdoba.
Ello indicaría que hay más de dos linajes de $A n$. triannulatus s.I. en Colombia, hipótesis que deberá corroborarse ampliando las recolecciones a otros departamentos donde se distribuye la especie y, también, aumentando el número de ejemplares.

En conclusión, según nuestros resultados, las poblaciones de An. triannulatus s.l. de Córdoba se comportan como una gran población, con un gran flujo de migrantes entre las localidades en estudio. 


\section{Conflicto de intereses}

Los autores declaran no tener conflictos de intereses.

\section{Financiación}

Este estudio fue financiado por el Programa de Investigación en Salud y Enfermedades Tropicales, Convenio 754-2013, entre el departamento de Córdoba y la Universidad del Sinú-Elías Bechara Zainum.

\section{Referencias}

1. Walter Reed Biosystematics Unit. Systematic catalog of Culicidae, 2017. Fecha de consulta: 16 de marzo de 2018. Disponible en: http://www.mosquitocatalog.org.

2. Faran M, Linthicum K. A handbook of the Amazonian species of Anopheles (Nyssorhynchus) (Diptera: Culicidae). Mosq Syst. 1981;13:1-81.

3. Chadde DD, Wilkerson R. Anopheles triannulatus (Neiva and Pinto): A new Anopheles record from Trinidad, West Indies. J Am Mosq Control Assoc. 2005;21:316-7. https:// doi.org/10.2987/8756-971X(2005)21[316:ATNAPA]2.0. $\mathrm{CO} ; 2$

4. González R, Carrejo N. Introducción al estudio taxonómico de Anopheles de Colombia: claves y notas de distribución. Segunda edicion. Cali: Universidad del Valle; 2009. p. 260.

5. Sinka M, Rubio-Palis Y, Manguin S, Patil A, Temperley W, Gething P, et al. The dominant Anopheles vectors of human malaria in the Americas: Occurrence data, distribution maps and bionomic précis. Parasit Vectors. 2010;3:72. https://doi. org/10.1186/1756-3305-3-72

6. Galardo AK, Arruda M, Couto AR, Wirtz R, Lounibos LP, Zimmerman RH. Malaria vector incrimination in three rural riverine villages in the Brazilian Amazon. Am J Trop Med Hyg. 2007;76:461-9. https://doi.org/10.4269/ajtmh.2007.76.461

7. Tadei WP, Thatcher BD. Malaria vectors in the Brazilian Amazon: Anopheles of the subgenus Nyssorhynchus. Rev Inst Med Trop São Paulo. 2000;42:87-94. https://doi. org/10.1590/S0036-46652000000200005

8. de Oliveira-Ferreira J, Lourenco-de-Oliveira R, Teva A, Deane LM, Daniel-Ribeiro CT. Natural malaria infections in Anophelines in Rondania State, Brazilian Amazon. Am J Trop Med Hyg. 1990;43:6-10.

9. Aramburú J, Ramal C, Witzig R. Malaria reemergence in the Peruvian Amazon Region. Emerg Infect Dis. 1999;5:209 15. https://doi.org/10.3201/eid0502.990204

10. Naranjo-Díaz N, Rosero DA, Rúa-Uribe G, Luckhart S, Correa MM. Abundance, behavior and entomological inoculation rates of anthropophilic anophelines from a primary Colombian malaria endemic area. Parasit Vectors. 2013;6:1-11. https://doi.org/10.1186/1756-3305-6-61

11. Rosero DN, Naranjo-Díaz N, Álvarez A, Cienfuegos C, Torres S, Luckhart S, et al. Colombian Anopheles triannulatus (Diptera: Culicidae) naturally infected with Plasmodium spp. ISRN Parasitol. 2013:2013:927453. https://doi.org/10.5402/2013/927453

12. Rosa-Freitas MG, Lourenço-de-Oliveira R, de CarvalhoPinto CJ, Flores-Mendoza C, Fernandes T, DoNascimiento S. Anopheline species complexes in Brazil.
Current knowledge of those related to malaria transmission. Mem Inst Oswaldo Cruz. 1998;93:651-5. https://doi. org/10.1590/S0074-02761998000500016

13. Silva-do-Nacimiento T, Lourenço-de-Oliveira R. Anopheles halophylus, a new species of the Subgenus Nyssorhynchus (Diptera: Culicidae) from Brazil. Mem Inst Oswaldo Cruz. 2002;97:801-11. https://doi.org/10.1590/S0074-0276200200 0600010

14. Rosa-Freitas MG, Tsouris P, Peterson AT, Honório NA, Barros FS, Aguiar DB, et al. An ecoregional classification for the state of Roraima, Brazil. The importance of landscape in malaria biology. Mem Inst Oswaldo Cruz. 2007;102:34957. https://doi.org/10.1590/S0074-02762007005000052

15. Silva-do-Nascimento TF, Wilkerson RC, Monteiro FA. Molecular confirmation of the specific status of Anopheles halophylus (Diptera: Culicidae) and evidence of a new cryptic species within An. triannulatus in Central Brazil. J Med Entomol. 2006;43:455-9.

16. Silva-do-Nascimento TF, Damazio L, Pitaluga R, Peixoto AA, Lourenço-de-Oliveira $\mathbf{R}$. Molecular divergence in the timeless and cpr genes among three sympatric cryptic species of the Anopheles triannulatus complex. Mem Inst Oswaldo Cruz. 2011;106:218-22. https://doi.org/10.1590/ S0074-02762011000900027

17. Brochero H, Pareja PX, Ortiz G, Olano VA. Sitios de cría y actividad de picadura de especies de Anopheles en el municipio de Cimitarra, Santander, Colombia. Biomédica. 2006; 26:269-77. https://doi.org/10.7705/biomedica.v26i2.1416

18. Gutiérrez LA, González JJ, Gómez GF, Castro MI, Rosero DA, Luckhart S, et al. Species composition and natural infectivity of anthropophilic Anopheles (Diptera: Culicidae) in Córdoba and Antioquia states in northwestern Colombia. Mem Inst Oswaldo Cruz. 2009;104:1117-24. https://doi. org/10.1590/S0074-02762009000800008

19. Gutiérrez LA, Naranjo NJ, Cienfuegos AV, Muskus CE, Luckhart S, Conn JE, et al. Population structure analyses and demographic history of the malaria vector Anopheles albimanus from the Caribbean and the Pacific regions of Colombia. Malar J. 2009;8:259. https://doi. org/10.1186/1475-2875-8-259

20. Rodríguez M, Pérez L, Caicedo JC, Prieto G, Arroyo JA, Kaur $\mathrm{H}$, et al. Composition and biting activity of Anopheles (Diptera: Culicidae) in the Amazon Region of Colombia. J Med Entomol. 2009;46:307-15.

21. Rosero DA, Jaramillo LM, Gutiérrez LA, Conn JE, Correa MM. Genetic diversity of Anopheles triannulatus s.l. (Diptera: Culicidae) from Northwestern and Southeastern Colombia. Am J Trop Med Hyg. 2012;87:910-20. https://doi. org/10.4269/ajtmh.2012.12-0285

22. Collins FH, Méndez A, Rasmussen MO, Mehaffey PC, Besansky NJ, Finnerty V. Ribosomal RNA gene probe differentiates member species of the Anopheles gambiae complex. Am J Trop Med Hyg. 1987;37:37-41. https://doi. org/10.4269/ajtmh.1987.37.37

23. Atencia M, Pérez M, Jaramillo M, Caldera S, Bejarano E. Primer reporte de la mutación F1534C asociada con resistencia cruzada a DDT y piretroides en Aedes aegypti en Colombia. Biomédica. 2016;36:432-7. https://doi.org/10. 7705/biomedica.v36i3.2834 
24. Folmer O, Black M, Hoeh W, Lutz R, Vrijenhoek R. DNA primers for amplification of mitochondrial cytochrome $\mathrm{C}$ oxidase subunit I from diverse metazoan invertebrates. Mol Mar Biol Biotechnol. 1994;3:294-9.

25. Kumar S, Stecher G, Tamura K. MEGA7: Molecular evolutionary genetics analysis version 7.0 for bigger datasets. Mol Biol Evol. 2016;33:1870-4. https://doi.org/10.1093/ molbev/msw054

26. Thompson J, Higgins D, Gibson T. CLUSTAL W: Improving the sensitivity of progressive multiple sequence alignment through sequence weighting, position-specific gap penalties and weight matrix choice. Nucleic Acids Res. 1994;22:4673-80.

27. Altschup S, Gish W, Miller W, Myers E, Lipman D. Basic local alignment search tool department of computer science. J Mol Biol. 1990;215:403-10. https://doi.org/10.1016/S00222836(05)80360-2

28. Ratnasingham S, Hebert PDN. Bold: The Barcode of Life Data System (http://www.barcodinglife.org). Molecular Ecology Notes. 2007;7:355-64. https://doi.org/10.1111/j.14718286.2007.01678.x

29. Librado P, Rozas J. DnaSP v5: A software for comprehensive analysis of DNA polymorphism data. Bioinformatics. 2009;25:1451-2. https://doi.org/10.1093/bioinformatics/btp187

30. Excoffier L, Lischer HE. Arlequin Suite ver 3.5: A new series of programs to perform population genetics analyses under Linux and Windows. Mol Ecol Resour. 2010;10:5647. https://doi.org/10.1111/j.1755-0998.2010.02847.x

31. Fluxus Technology. Network 5.0. Clare: Fluxus Technology Ltd.; 2017.

32. Badelt H, Forster P, Rohl A. Median-joining networks for inferring intraspecific phylogenies. Mol Biol Evol. 1999;16: 37-48. https://doi.org/10.1093/oxfordjournals.molbev.a026036

33. Posada D. jModelTest: Phylogenetic model averaging. Mol Biol Evol. 2008;25:1253-6. https://doi.org/10.1093/molbev/ msn083

34. Ronquist F, Huelsenbeck J. MrBayes 3: Bayesian phylogenetic inference under mixed models. Bioinformatics. 2003; 19:1572-4. https://doi.org/10.1093/bioinformatics/btg180
35. Gutiérrez LA, Gómez GF, González JJ, Castro MI, Luckhart S, Conn JE, et al. Microgeographic genetic variation of the malaria vector Anopheles darlingi Root (Diptera: Culicidae) from Córdoba and Antioquia, Colombia. Am J Trop Med Hyg. 2010;83:38-47. https://doi.org/10.4269/ ajtmh.2010.09-0381

36. Jaramillo LM, Gutiérrez LA, Luckhart S, Conn JE, Correa MM. Molecular evidence for a single taxon, Anopheles nuneztovari s.l., from two endemic malaria regions in Colombia. Mem Inst Oswaldo Cruz. 2011;106:1017-23. https://doi.org/10.1590/S0074-02762011000800020

37. Pedro PM, Uezu A, Sallum MA. Concordant phylogeographies of 2 malaria vectors attest to common spatial and demographic histories. J Hered. 2010;101:618-27. https:// doi.org/10.1093/jhered/esq054

38. Urrea PA, Correa M, Naranjo-Díaz N. Variabilidad genética de Anopheles punctimacula s.l. en dos localidades de la zona endémica para la malaria: el Bajo Cauca y Alto Sinú. Hechos Microbiológicos. 2014;5:51-62.

39. Dai $Y$, Huang $X$, Cheng $P$, Liu L, Wang $H$, Wang $H$, et al. Development of insecticide resistance in malaria vector Anopheles sinensis populations from Shandong province in China. Malar J. 2015;14:62. https://doi.org/10.1186/s12936015-0592-8

40. Scarpassa VM, Cunha-Machado AS, Saraiva JF. Evidence of new species for malaria vector Anopheles nuneztovari sensu lato in the Brazilian Amazon region. Malar J. 2016;15:205. https://doi.org/10.1186/s12936-0161217-6

41. McKeon SN, Lehr M, Wilkerson RC, Ruiz JF, Sallum $\mathbf{M}$, Lima JB, et al. Lineage divergence detected in the malaria vector Anopheles marajoara (Diptera: Culicidae) in Amazonian Brazil. Malar J. 2010;9:271. https://doi. org/10.1186/1475-2875-9-271

42. Ahumada ML, Orjuela LI, Pareja PX, Conde M, Cabarcas DM, Cubillos EFG, et al. Spatial distributions of Anopheles species in relation to malaria incidence at 70 localities in the highly endemic Northwest and South Pacific coast regions of Colombia. Malar J. 2016;15:407. https://doi.org/10.1590/ S0074-02762009000800008 